\title{
Square-integrability of solutions of the Yamabe equation
}

\author{
Bernd Ammann, Mattias Dahl and Emmanuel Humbert
}

We show that solutions of the Yamabe equation on certain $n$-dimensional non-compact Riemannian manifolds, which are bounded and $L^{p}$ for $p=2 n /(n-2)$ are also $L^{2}$. This $L^{p}-L^{2}$ implication provides explicit constants in the surgery-monotonicity formula for the smooth Yamabe invariant in our paper [4]. As an application we see that the smooth Yamabe invariant of any twoconnected compact seven-dimensional manifold is at least 74.5. Similar conclusions follow in dimension 8 and in dimensions $\geq 11$.

1 Introduction $\quad 892$

$2 \quad$ Preliminaries and notation $\quad 893$

3 Main theorem $\quad 898$

4 A counterexample to Theorem 3.1 for $k=n-3 \quad 905$

5 Consequences for the surgery formula 906

$\begin{array}{lll}6 & \text { Topological applications } & 907\end{array}$

$\begin{array}{ll}\text { Appendix A Details of an argument } & 912\end{array}$

$\begin{array}{lr}\text { Acknowledgments } & 914\end{array}$

$\begin{array}{ll}\text { References } & 914\end{array}$ 


\section{Introduction}

The goal of this paper is to show that bounded positive solutions of the Yamabe equation on certain $n$-dimensional non-compact Riemannian manifolds $\mathbb{M}_{c}^{n, k}$ with finite $L^{p_{n}}$-norm, $p_{n}=2 n /(n-2)$, also have finite $L^{2}$-norm. For $c \neq 0$ these spaces $\mathbb{M}_{c}^{n, k}$ are products of rescaled hyperbolic space with a standard sphere, while $\mathbb{M}_{0}^{n, k}=\mathbb{R}^{k+1} \times \mathbb{S}^{n-k-1}$. The integer $k$ satisfies $0 \leq$ $k \leq n-3$. The goal is achieved in some cases, we then say that the $L^{p_{n}}-L^{2}-$ implication holds. In particular, it holds for $0 \leq k \leq n-4$. If $k=n-3$ and if $n$ is sufficiently large we will find counterexamples; see Section 4.

In the favourable cases, the proof of the $L^{p_{n}}-L^{2}$-implication is obtained by a combination of tricky integration and suitable estimates, and there is not much hope to generalize our technique to a much larger class of noncompact manifolds. The reader will thus probably ask why we develop such estimates for these special spaces.

The reason is that these spaces appear naturally as limit spaces in the surgery construction of our paper [4], and this limit construction also provides $L^{p_{n}}$-solutions of the Yamabe equation. The main result of [4] is a surgery formula for the smooth Yamabe invariant $\sigma(M)$; see Section 2.3 for the definition. The surgery formula states that if $M$ is a compact manifold of dimension $n$ and if $N$ is obtained from $M$ through $k$-dimensional surgery, then the smooth Yamabe invariants $\sigma(M)$ and $\sigma(N)$ satisfy

$$
\sigma(N) \geq \min \left(\sigma(M), \Lambda_{n, k}\right)
$$

provided that $k \leq n-3$. The definition of the numbers $\Lambda_{n, k}$ is quite involved; see Section 2.6, but they are proven to be positive and to depend only on $n$ and $k$. The $L^{p_{n}}-L^{2}$-implication helps to derive explicit lower bounds for these numbers; see Sections 2.4 to 2.6.

Using estimates for product manifolds from the article [3] we obtain explicit positive lower bounds for $\Lambda_{n, k}$ in the case $2 \leq k \leq n-4$; see Corollary 5.1 for details. This leads directly to a uniform positive lower bound for the smooth Yamabe invariant of two-connected manifolds that are boundaries of compact spin manifolds; see Corollary 6.3. In dimensions $n \in\{7,8\}$ and $n \geq 11$ we obtain an explicit positive lower bound $\sigma(M)$, provided that $M$ is two-connected with vanishing index $\alpha(M) \in K O_{n}(\mathrm{pt})$; see Corollary 6.7. Note that $S^{5}$ is the only two-connected closed five-manifold. Further, a two-connected closed 6-manifold is diffeomorphic to the connected sum of a number of copies of $S^{3} \times S^{3}$ and the smooth Yamabe invariant can be 
estimated using results of [8] and [3]. It is also known that $\alpha(M) \neq 0$ implies $\sigma(M)=0$.

Using results from $[2,11,12]$ an explicit positive lower bound can also be obtained in the cases $(n, k) \in\{(4,1),(5,1),(5,2),(6,3),(9,1),(10,1)\}$, see [2] for details about these dimensions.

\section{Preliminaries and notation}

\subsection{Spaces of constant curvature and the model spaces $\mathbb{M}_{c}^{n, k}$}

Here we fix notation for the spaces of constant curvature and we define the spaces $\mathbb{M}_{c}^{n, k}$.

We denote the Euclidean metric on $\mathbb{R}^{n}$ by $\xi^{n}$. The sphere $S^{n} \subset \mathbb{R}^{n+1}$ equipped with its standard round metric $\rho^{n}$ is denoted by $\mathbb{S}^{n}$. We set $\omega_{\ell}:=\operatorname{vol}\left(\mathbb{S}^{\ell}\right)$. For $c \in \mathbb{R}$ let $\mathbb{H}_{c}^{k+1}$ be the simply connected, connected, complete Riemannian manifold of constant sectional curvature $-c^{2}$. Its metric will be denoted as $\eta_{c}^{k+1}$. Polar coordinates around a point $x_{0}$ gives an identification of $\mathbb{H}_{c}^{k+1} \backslash\left\{x_{0}\right\}$ with $(0, \infty) \times S^{k}$ under which

$$
\eta_{c}^{k+1}=d r^{2}+\operatorname{sh}_{c}(r)^{2} \rho^{k}
$$

where

$$
\operatorname{sh}_{c}(r):= \begin{cases}\frac{1}{c} \sinh (c r) & \text { if } c \neq 0 \\ r & \text { if } c=0\end{cases}
$$

We denote the product metric on $\mathbb{H}_{c}^{k+1} \times \mathbb{S}^{n-k-1}$ by

$$
G_{c}:=\eta_{c}^{k+1}+\rho^{n-k-1}
$$

and we define the model space $\mathbb{M}_{c}^{n, k}$ through

$$
\mathbb{M}_{c}^{n, k}:=\mathbb{H}_{c}^{k+1} \times \mathbb{S}^{n-k-1}
$$

The scalar curvature of $\mathbb{M}_{c}^{n, k}$ is $s^{G_{c}}=-c^{2} k(k+1)+(n-k-1)(n-k-2)$. Note that $\mathbb{M}_{c}^{n, k}=\mathbb{M}_{-c}^{n, k}$. As a consequence all following infima over $c \in[-1,1]$ could be taken over $c \in[0,1]$. From the conformal point of view the case $c= \pm 1$ is special since $\mathbb{M}_{ \pm 1}^{n, k}=\mathbb{H}_{ \pm 1}^{k+1} \times \mathbb{S}^{n-k-1}$ is conformal to $\mathbb{S}^{n} \backslash \mathbb{S}^{k}$; see [4, Proposition 3.1]. 


\subsection{The conformal Yamabe constant}

For integers $n \geq 3$ we set $a_{n}:=\frac{4(n-1)}{n-2}$ and $p_{n}:=\frac{2 n}{n-2}$. For a Riemannian manifold $(M, g)$ we denote the scalar curvature by $s^{g}$, the Laplace operator $\Delta^{g}$, and the volume form $d v^{g}$. The conformal Laplacian is defined as $L^{g}:=$ $a_{n} \Delta^{g}+s^{g}$. In general the dependence on the Riemannian metric is indicated by adding the metric as a superscript.

Let $C_{c}^{\infty}(M)$ denote the space of compactly supported smooth functions on $M$. For a Riemannian manifold $(M, g)$ of dimension $n \geq 3$ we define the Yamabe functional by

$$
\mathcal{F}^{g}(u):=\frac{\int_{M}\left(a_{n}|d u|_{g}^{2}+s^{g} u^{2}\right) d v^{g}}{\left(\int_{M}|u|^{p_{n}} d v^{g}\right)^{\frac{2}{p_{n}}}}
$$

where $u \in C_{c}^{\infty}(M)$ does not vanish identically. The conformal Yamabe constant $\mu(M, g)$ of $(M, g)$ is defined by

$$
\mu(M, g):=\inf _{u \in C_{c}^{\infty}(M), u \neq 0} \mathcal{F}^{g}(u)
$$

Here $M$ is allowed to be compact or non-compact.

If $M$ is compact, then the infimum is attained by a positive smooth function. It thus satisfies the associated Euler-Lagrange equation, called the Yamabe equation,

$$
L^{g} u=\mu u^{p_{n}-1}
$$

for a suitable constant $\mu$. The invariant is actually a conformal invariant: if $g_{1}$ is conformal to $g_{2}$, then $\mu\left(M, g_{1}\right)=\mu\left(M, g_{2}\right)$.

\subsection{The smooth Yamabe invariant}

Let $M$ be a compact manifold of dimension $n \geq 3$. The smooth Yamabe invariant of $M$ defined as

$$
\sigma(M):=\sup \mu(M, g)
$$

where the supremum is taken over the set of all Riemannian metrics on $M$. It is known that $\sigma\left(S^{n}\right)=\mu\left(\mathbb{S}^{n}\right)=n(n-1) \omega_{n}^{2 / n}$. 


\subsection{The role of the model spaces in the surgery formula}

To give some background and motivation we will now briefly explain the role of the model spaces $\mathbb{M}_{c}^{n, k}$ in [4], and we want to give a rough idea why the invariants $\Lambda_{n, k}$ defined in the following subsections appear. The discussion in the present subsection is not needed in the proofs of the following results. We thus try to avoid technical details, and do not aim for logical completeness.

Assume that $(M, g)$ is a compact Riemannian manifold of dimension $n \geq 3$ and that $N$ is obtained by $k$-dimensional surgery from $M$, where $0 \leq k \leq n-3$. In [4] a sequence of metrics $g_{i}$ is constructed on $N$. To prove the surgery formula (1.1) we show

$$
\limsup _{i \rightarrow \infty} \mu\left(N, g_{i}\right) \geq \min \left(\mu(M, g), \Lambda_{n, k}\right)
$$

for a suitable positive constant $\Lambda_{n, k}$.

The solution of the Yamabe problem on $\left(N, g_{i}\right)$ provides positive smooth functions $u_{i} \in C^{\infty}(N)$ satisfying the Yamabe equation

$$
L^{g_{i}} u=\mu_{i} u_{i}^{p_{n}-1}
$$

where $\mu_{i}=\mu\left(N, g_{i}\right)$ and $\left\|u_{i}\right\|_{L^{p_{n}}}=1$.

In order to prove (2.2), one analyses the "limits" of the functions $u_{i}$ in various cases. In some cases, these functions $u_{i}$ "converge" to a nontrivial solution of the Yamabe equation $L^{g} u=\mu u^{p_{n}-1}$ on $(M, g)$ with $\mu=$ $\limsup \mu_{i}$, and it follows that $\lim \sup _{i \rightarrow \infty} \mu\left(N, g_{i}\right) \geq \mu(M, g)$. In other cases the functions concentrate in some points, and $\lim \sup _{i \rightarrow \infty} \mu\left(N, g_{i}\right) \geq \mu\left(\mathbb{S}^{n}\right)$ follows by "blowing-up" such points. This means that one suitably rescales normal coordinates centred in the blow-up point, and then the solutions of the Yamabe equation converge to a solution on $\mathbb{R}^{n}$, which then yields a solution of the Yamabe equation on a sphere. This phenomenon is also often described in the literature by saying that "a sphere bubbles off".

However, it can also happen that the functions $u_{i}$ converge to a solution of the Yamabe equation on a model space $\mathbb{M}_{c}^{n, k}$ with $|c| \leq 1$, this corresponds to Subcases II.1.1 and II.2 in the proof of Theorem 6.1 in [4]. In this case one obtains points $x_{i} \in N$, such that the pointed Riemannian manifolds $\left(N, g_{i}, x_{i}\right)$ "converge" to $\left(\mathbb{M}_{c}^{n, k}, \bar{x}\right)$. Here "convergence" means that balls of arbitrary radius $R$ around $x_{i}$ in $\left(N, g_{i}\right)$ converge for fixed $R$ and $i \rightarrow \infty$ in the $C^{\infty}$-sense to a ball of radius $R$ around $\bar{x}$ in $\mathbb{M}_{c}^{n, k}$. The functions $u_{i}$ will then converge to a positive solution $\bar{u}$ of the Yamabe equation (2.1) on $\mathbb{M}_{c}^{n, k}$. The $L^{p_{n}}$-norm of the limit function does not increase, that is $\|\bar{u}\|_{L^{p_{n}}} \leq 1$. 
This raises the following question.

Question 2.1. Assume that $\bar{u} \in C^{\infty}\left(\mathbb{M}_{c}^{n, k}\right)$ is a positive solution of the Yamabe equation $L^{G_{c}} \bar{u}=\lambda \bar{u}^{p_{n}-1}$ with $0<\|\bar{u}\|_{L^{p_{n}}} \leq 1$. Does this imply $\lambda \geq \mu\left(\mathbb{M}_{c}^{n, k}\right)$ ?

If $\bar{u}$ is in $L^{2}$, then it is already in the Sobolev space $H^{1,2}$ and integration by parts $\int \bar{u} \Delta \bar{u} d v=\int|d \bar{u}|^{2} d v$ is allowed on $\mathbb{M}_{c}^{n, k}$, see Appendix A. It then easily follows that that the answer to the question is positive. In this case we will say that the $L^{p_{n}}-L^{2}$-implication holds.

In turn the $L^{p_{n}}-L^{2}$-implication implies that (2.2) holds for

$$
\Lambda_{n, k}:=\inf _{c \in[-1,1]} \mu\left(\mathbb{M}_{c}^{n, k}\right) .
$$

We will find conditions under which the $L^{p_{n}}-L^{2}$-implication holds. But we will also obtain examples where it is violated, see Section 4.

The fact that the $L^{p_{n}}-L^{2}$-implication is violated in some cases led to a technical difficulty in [4] which was solved by introducing the constant $\Lambda_{n, k}^{(2)}$ into the definition of $\Lambda_{n, k}$ in [4, Definition 3.2]. This is sufficient for proving the positivity of the constant $\Lambda_{n, k}$. However, in order to obtain an explicit positive lower bound for $\Lambda_{n, k}$ one would like to avoid the constant $\Lambda_{n, k}^{(2)}$. The possibility to prove the $L^{p_{n}}-L^{2}$-implication in some cases was mentioned in [4, Remark 3.4]. As a consequence finding a positive lower bound for $\Lambda_{n, k}$ reduces to finding a positive lower bound for the constants $\mu\left(\mathbb{M}_{c}^{n, k}\right)$, uniform in $c \in[0,1]$.

In the meantime new results for the explicit lower bounds for $\mu\left(\mathbb{M}_{c}^{n, k}\right)$ were obtained in $[2,3]$, and a proof of $[4$, Remark 3.4] is needed. The goal of the present article is to provide this proof.

\subsection{Modified conformal Yamabe constants}

The technical difficulty described in the previous subsection required the introduction of a modified conformal Yamabe constant. In fact, two different subcases require two versions of modified constants, namely the modified conformal Yamabe constants $\mu^{(1)}(N, h)$ and $\mu^{(2)}(N, h)$, defined below. Our article aims to give some clarification of the relation between these invariants for the model spaces $\mathbb{M}_{c}^{n, k}$.

Let $(N, h)$ be a Riemannian manifold of dimension $n$. For $i=1,2$ we let $\Omega^{(i)}(N, h)$ be the set of non-negative $C^{2}$ functions $u$ on $N$, which solve the 
Yamabe equation

$$
L^{h} u=\mu u^{p_{n}-1}
$$

for some $\mu=\mu(u) \in \mathbb{R}$. We also require that the functions $u \in \Omega^{(i)}(N, h)$ satisfy
(a) $u \not \equiv 0$,
(b) $\|u\|_{L^{p_{n}(N)}} \leq 1$,
(c) $u \in L^{\infty}(N)$,

together with

(d1) $u \in L^{2}(N)$, for $i=1$, or

(d2) $\mu(u)\|u\|_{L^{\infty}(N)}^{p_{n}-2} \geq \frac{(n-k-2)^{2}(n-1)}{8(n-2)}$, for $i=2$.

For $i=1,2$ we set

$$
\mu^{(i)}(N, h):=\inf _{u \in \Omega^{(i)}(N, h)} \mu(u) .
$$

In particular $\mu^{(i)}(N, h)=\infty$ if $\Omega^{(i)}(N, h)$ is empty. If $N$ is compact then the solution of the Yamabe problem trivially implies $\mu(N, h)=\mu^{(1)}(N, h) \leq$ $\mu^{(2)}(N, h)$.

We will use this for $(N, h)=\mathbb{M}_{c}^{n, k}$. In [4, Lemma 3.5] we already showed $\mu^{(1)}\left(\mathbb{M}_{c}^{n, k}\right) \geq \mu\left(\mathbb{M}_{c}^{n, k}\right)$ if $0 \leq k \leq n-3$. In the present paper, we will show that (b) implies (d1) in many cases. As a consequence we will obtain

$$
\mu^{(2)}\left(\mathbb{M}_{c}^{n, k}\right) \geq \mu^{(1)}\left(\mathbb{M}_{c}^{n, k}\right) \geq \mu\left(\mathbb{M}_{c}^{n, k}\right)
$$

in theses cases. We refer to Theorem 3.1 and Corollary 3.2 for details.

\subsection{The numbers $\Lambda_{n, k}$}

For integers $n \geq 3$ and $0 \leq k \leq n-3$ set

$$
\Lambda_{n, k}^{(i)}:=\inf _{c \in[-1,1]} \mu^{(i)}\left(\mathbb{M}_{c}^{n, k}\right)
$$

and

$$
\Lambda_{n, k}:=\min \left\{\Lambda_{n, k}^{(1)}, \Lambda_{n, k}^{(2)}\right\}
$$

It is not hard to show that $\Lambda_{n, 0}=\mu\left(\mathbb{S}^{n}\right)$; see [4, Section 3.5]. The following positivity result for $\Lambda_{n, k}$ is proved in [4, Theorem 3.3]. 
Theorem 2.2. For all $n \geq 3$ and $0 \leq k \leq n-3$, we have $\Lambda_{n, k}>0$.

Furthermore, the following surgery result is concluded in [4, Corollary 1.4].

Theorem 2.3. Inequality (1.1) holds for $0 \leq k \leq n-3$ and the numbers $\Lambda_{n, k}>0$ defined above.

\section{Main theorem}

Theorem 3.1. Let $c \in[-1,1]$ and let $u \in L^{\infty}\left(\mathbb{M}_{c}^{n, k}\right) \cap L^{p_{n}}\left(\mathbb{M}_{c}^{n, k}\right) \cap$ $C^{2}\left(\mathbb{M}_{c}^{n, k}\right)$ be a uniformly continuous, positive solution of

$$
L^{G_{c}} u \leq \mu u^{p_{n}-1}
$$

Assume that

$$
2 k|c|<n(n-k-2),
$$

then $u \in L^{2}\left(\mathbb{M}_{c}^{n, k}\right)$.

Inequality (3.2) holds when

- $n \leq 5, k \in\{0, \ldots, n-3\}$, and $|c| \leq 1$; or

- $n \geq 6, k \in\{0, \ldots, n-4\}$, and $|c| \leq 1$; or

- $n=6, k=n-3$, and $|c|<1$.

This follows from the fact that $2 k \leq n(n-k-2)$ is equivalent to $k \leq n-4+\frac{8}{n+2}$.

Non-negative $L^{\infty}$-solutions of (2.3) on manifolds with bounded geometry will be uniformly continuous due to standard local regularity and Sobolev embeddings (one even can show boundedness in $C^{3, \alpha}$ for small $\alpha>0$ ). Thus, the conditions in Theorem 3.1 are satisfied for all $L^{\infty}$-solutions of $(2.3)$ on $\mathbb{M}_{c}^{n, k}$, in particular for all $u \in \Omega^{(i)}\left(\mathbb{M}_{c}^{n, k}\right), i=1,2$.

Also note that a non-negative solution of (2.3) will be either identically zero or everywhere positive, due to the maximum principle.

Remark. We expect that a similar theorem for weak solutions of (3.1) holds as well. Such a version for weak solutions is required in current work [5] of Nadine Große in collaboration with the first author. This project will 
relate the spinorial analogue of $\Lambda_{n, k}$ defined in [1] to the constants $\Lambda_{n, k}$ defined above.

More precisely, we expect that Theorem 3.1 also holds for uniformly continuous, non-negative functions $u \in L^{\infty}\left(\mathbb{M}_{c}^{n, k}\right) \cap L^{p_{n}}\left(\mathbb{M}_{c}^{n, k}\right)$ satisfying (3.1) in the distributional sense, without the conditions of positivity and $u \in C^{2}$. Unfortunately the proof presented below is no longer valid in the distributional setting, as square roots of distributions and products of distributions would appear and it remains unclear how to handle these objects.

However, we worked out a distributional version of the proof below for non-negative functions $u \in L^{\infty}\left(\mathbb{M}_{c}^{n, k}\right) \cap L^{p_{n}}\left(\mathbb{M}_{c}^{n, k}\right)$ that are $C^{2}$ on $\Omega$ for $\Omega:=\left\{x \in \mathbb{M}_{c}^{n, k} \mid u(x)>0\right\}$, that satisfy (3.1) on $\Omega$, and that satisfy $u^{2} \in C^{1}\left(\mathbb{M}_{c}^{n, k}\right)$. Under the above conditions on $c, n$ and $k$, we then get the conclusion $u \in L^{2}\left(\mathbb{M}_{c}^{n, k}\right)$, as well. The non- $C^{2}$ functions in [5] satisfy all these conditions. In the proof of the generalized version of Theorem 3.1, one has to replace integrals over $F_{r}$ by integrals over $\left\{x \in F_{r} \mid u(x) \geq \varepsilon\right\}$. The boundary terms thus obtained can be estimated by 0 .

Corollary 3.2. We have

$$
\mu^{(2)}\left(\mathbb{M}_{c}^{n, k}\right) \geq \mu\left(\mathbb{M}_{c}^{n, k}\right)
$$

for all $k \leq n-4$ and $|c| \leq 1$. The same statement holds for $k=n-3$, $n \in\{4,5\}$ and $|c| \leq 1$. And it also holds for $k=3, n=6,|c|<1$.

Proof of Corollary 3.2. Under the conditions of the corollary Assumption (3.2) holds, and hence Theorem 3.1 implies

$$
\Omega^{(2)}\left(\mathbb{M}_{c}^{n, k}\right) \subset \Omega^{(1)}\left(\mathbb{M}_{c}^{n, k}\right)
$$

and as a consequence we get

$$
\mu^{(2)}\left(\mathbb{M}_{c}^{n, k}\right) \geq \mu^{(1)}\left(\mathbb{M}_{c}^{n, k}\right)
$$

On the other hand, it is proved in [4, Lemma 3.5] that

$$
\mu^{(1)}\left(\mathbb{M}_{c}^{n, k}\right) \geq \mu\left(\mathbb{M}_{c}^{n, k}\right)
$$

for all $k \in\{0, \ldots, k-3\}$. The corollary follows.

Proof of Theorem 3.1. We will now give the proof of Theorem 3.1 in five steps. Let $u$ be as in the statement of this theorem.

Step 1. The function $u$ tends to 0 at infinity. 
We proceed by contradiction and assume that there is an $\varepsilon>0$ and a sequence of points $\left(x_{j}\right)_{j \in \mathbb{N}}$ tending to infinity (as a sequence in $j$ ) such that $u\left(x_{j}\right) \geq \varepsilon$. Denote by $\overline{B(x, 1)}$ the closed ball of radius 1 around a point $x$. By taking a subsequence of $\left(x_{j}\right)$ which tends fast enough to infinity, we can assume that the balls $\overline{B\left(x_{j}, 1\right)}$ are all disjoint. Since $u$ is in $L^{p_{n}}$ we have

$$
\lim _{j \rightarrow \infty} \int_{\overline{B\left(x_{j}, 1\right)}} u^{p_{n}} d v^{G_{c}}=0 .
$$

Since $\mathbb{M}_{c}^{n, k}$ is homogenous, there are isometries $\varphi_{j}: \overline{B\left(x_{j}, 1\right)} \rightarrow \overline{B(O, 1)}$ where $O$ is any fixed point in $\mathbb{M}_{c}^{n, k}$. Obviously $v_{j}:=u \circ \varphi_{j}^{-1}$ satisfy

$$
\lim _{j \rightarrow \infty} \int_{\overline{B(O, 1)}} v_{j}^{p_{n}} d v^{G_{c}}=0
$$

and

$$
v_{j}(O) \geq \varepsilon .
$$

Since $u$ is assumed to be uniformly continuous and bounded, the sequence $v_{j}$ is equicontinuous and bounded. A subsequence of $\left(v_{j}\right)$ converges uniformly, i.e., in $C^{0}(\overline{B(O, 1)}, \mathbb{R})$, to a continuous function $v \in C^{0}(\overline{B(O, 1)}, \mathbb{R})$.

Finally, (3.3) and (3.4) tell us that

$$
\int_{\overline{B(O, 1)}} v^{p_{n}} d v^{G_{c}}=0
$$

and

$$
v(O) \geq \varepsilon,
$$

which gives the desired contradiction. This ends the proof of Step 3.

We now work in polar coordinates on the hyperbolic space factor of $\mathbb{M}_{c}^{n, k}=\mathbb{H}_{c}^{k+1} \times \mathbb{S}^{n-k-1}$ as introduced in Section 2.1. We thus study the metric $G_{c}=d r^{2}+\operatorname{sh}_{c}(r)^{2} \rho^{k}+\rho^{n-k-1}$ on the manifold $(0, \infty) \times S^{k} \times S^{n-k-1}$. Using these coordinates, we denote by $F_{r}$ the set of constant $r$, that is $F_{r}:=S^{k} \times S^{n-k-1}$ and we denote the restriction of $g$ to $F_{r}$ by $g_{r}=\operatorname{sh}_{c}(r)^{2} \rho^{k}+\rho^{n-k-1}$. We define

$$
\omega(r):=\left(\int_{F_{r}} u^{2} d v^{g_{r}}\right)^{\frac{1}{2}},
$$

for $r>0$. Next, we prove a differential inequality for $\omega$. 
Step 2. For any $\gamma$ with $0<\gamma<\frac{n-k-2}{2}$, there is an $r_{0}(\gamma, u)$ such that

$$
\omega^{\prime \prime}(r) \geq \gamma^{2} \omega(r)
$$

for all $r>r_{0}(\gamma, u)$.

The argument for this step is a modification of the proof of Theorem 5.2 in $[4]$.

The Laplacian operators of the total space $\Delta^{G_{c}}$ and of the fibres $\Delta^{g_{r}}$ are related by

$$
\Delta^{G_{c}}=\Delta^{g_{r}}-\partial_{r}^{2}+(n-1) H_{r} \partial_{r},
$$

where $H_{r}$ denotes the mean curvature of the fibre $F_{r}$ in $\mathbb{M}_{c}^{n, k}$. It follows that

$$
\begin{aligned}
\int_{F_{r}} u \Delta^{G_{c}} u d v^{g_{r}} & =\int_{F_{r}}\left(u \Delta^{g_{r}} u-u \partial_{r}^{2} u+(n-1) H_{r} u \partial_{r} u\right) d v^{g_{r}} \\
& =\int_{F_{r}}\left(\left|d_{\mathrm{vert}} u\right|^{2}-u \partial_{r}^{2} u+(n-1) H_{r} u \partial_{r} u\right) d v^{g_{r}},
\end{aligned}
$$

where $d_{\text {vert }} u$ denotes the differential of $u$ along the fibre, that is $d_{\text {vert }} u=$ $d\left(u_{\mid F_{r}}\right)$. Using Inequality (3.1) we get

$$
a_{n} \int_{F_{r}}\left(u \partial_{r}^{2} u-(n-1) H_{r} u \partial_{r} u\right) d v^{g_{r}} \geq s^{G_{c}} \omega(r)^{2}-\mu \int_{F_{r}} u^{p_{n}} d v^{g_{r}}
$$

Computing the derivative of $\omega(r)^{2} / 2$ we get

$$
\begin{aligned}
\omega^{\prime}(r) \omega(r) & =\frac{1}{2} \frac{d}{d r} \int_{F_{r}} u^{2} d v^{g_{r}} \\
& =\int_{F_{r}} u \partial_{r} u d v^{g_{r}}-\frac{n-1}{2} H_{r} \omega(r)^{2},
\end{aligned}
$$

where we used that $H_{r}$ is constant on $F_{r}$. Differentiating this again and using Inequality (3.5) we get

$$
\begin{aligned}
\omega^{\prime}(r)^{2}+\omega^{\prime \prime}(r) \omega(r)= & \int_{F_{r}}\left(\partial_{r} u\right)^{2} d v^{g_{r}}+\int_{F_{r}}\left(u \partial_{r}^{2} u-(n-1) H_{r} u \partial_{r} u\right) d v^{g_{r}} \\
& -\frac{n-1}{2}\left(\partial_{r} H_{r}\right) \omega(r)^{2}-(n-1) H_{r} \omega^{\prime}(r) \omega(r) \\
\geq & \int_{F_{r}}\left(\partial_{r} u\right)^{2} d v^{g_{r}}+\frac{s^{G_{c}}}{a_{n}} \omega(r)^{2}-\frac{\mu}{a_{n}} \int_{F_{r}} u^{p_{n}} d v^{g_{r}} \\
& -\frac{n-1}{2}\left(\partial_{r} H_{r}\right) \omega(r)^{2}-(n-1) H_{r} \omega^{\prime}(r) \omega(r) .
\end{aligned}
$$


From the Cauchy-Schwarz inequality, we get

$$
\begin{aligned}
\omega(r)^{2} \int_{F_{r}}\left(\partial_{r} u\right)^{2} d v^{g_{r}} & \geq\left(\int_{F_{r}} u\left(\partial_{r} u\right) d v^{g_{r}}\right)^{2} \\
& =\left(\omega^{\prime}(r) \omega(r)+\frac{n-1}{2} H_{r} \omega(r)^{2}\right)^{2}
\end{aligned}
$$

where we used Equation (3.6) in the second line. Thus,

$$
\int_{F_{r}}\left(\partial_{r} u\right)^{2} d v^{g_{r}} \geq\left(\omega^{\prime}(r)+\frac{n-1}{2} H_{r} \omega(r)\right)^{2} .
$$

Let $\varepsilon>0$ be a constant to be fixed later. By Step 3 we have

$$
\frac{\mu}{a_{n}} \int_{F_{r}} u^{p_{n}} d v^{g_{r}} \leq \varepsilon \omega(r)^{2}
$$

for all $r$ large enough (depending on $\varepsilon$ ). Inserting (3.8) and (3.9) into (3.7) we obtain

$$
\begin{aligned}
\omega^{\prime}(r)^{2}+\omega^{\prime \prime}(r) \omega(r) \geq & \left(\omega^{\prime}(r)+\frac{n-1}{2} H_{r} \omega(r)\right)^{2}+\frac{s^{G_{c}}}{a_{n}} \omega(r)^{2}-\varepsilon \omega(r)^{2} \\
& -\frac{n-1}{2}\left(\partial_{r} H_{r}\right) \omega(r)^{2}-(n-1) H_{r} \omega^{\prime}(r) \omega(r),
\end{aligned}
$$

or after some rearranging,

$$
\omega^{\prime \prime}(r) \geq \underbrace{\left(\frac{(n-1)^{2}}{4} H_{r}^{2}+\frac{s^{G_{c}}}{a_{n}}-\varepsilon-\frac{n-1}{2}\left(\partial_{r} H_{r}\right)\right)}_{=: \alpha(r)} \omega(r) .
$$

A computation tells us that

$$
H_{r}=-\frac{k}{n-1} \partial_{r} \ln \operatorname{sh}_{c}(r)= \begin{cases}-\frac{k}{n-1} c \operatorname{coth}(c r) & \text { if } c \neq 0 \\ -\frac{k}{n-1} \frac{1}{r} & \text { if } c=0\end{cases}
$$

so in particular,

$$
\lim _{r \rightarrow \infty} H_{r}=-\frac{k}{n-1}|c|
$$

and

$$
\lim _{r \rightarrow \infty} \partial_{r} H_{r}=0 .
$$


Using (3.11) and (3.12) together with $s^{G_{c}}=-c^{2} k(k+1)+(n-k-1)$ $(n-k-2)$, we see that the coefficient $\alpha(r)$ in the right-hand side in (3.10) tends to $\alpha_{c}-\varepsilon$ where

$$
\begin{aligned}
\alpha_{c} & :=\frac{(n-1)^{2}}{4} \frac{k^{2}}{(n-1)^{2}} c^{2}+\frac{n-2}{4(n-1)}\left(-c^{2} k(k+1)+(n-k-1)(n-k-2)\right) \\
& =-(n-k-2) \frac{k}{4(n-1)} c^{2}+\frac{(n-2)(n-k-1)(n-k-2)}{4(n-1)} \\
& \geq-(n-k-2) \frac{k}{4(n-1)}+\frac{(n-2)(n-k-1)(n-k-2)}{4(n-1)} \\
& =\frac{(n-k-2)^{2}}{4} .
\end{aligned}
$$

Here the inequality comes from the fact that the coefficient of $c^{2}$ is negative, so the smallest value over $c \in[-1,1]$ is attained for $c=1$. Choosing $\varepsilon$ small enough we have proved Step 3.

By assumption $u$ and thus $\omega$ are positive. As a consequence we can define

$$
\tau(r):=(\ln \omega(r))^{\prime}
$$

for $r>0$.

Step 3. One of the following statements is true,

$$
\begin{gathered}
\liminf _{r \rightarrow \infty} \tau(r) \geq(n-k-2) / 2, \\
\limsup _{r \rightarrow \infty} \tau(r) \leq-(n-k-2) / 2 .
\end{gathered}
$$

Assume that $(3.13 \mathrm{~b})$ is not true. Then there is a $\tilde{r} \geq r_{0}(\gamma)$ with $\tau(\tilde{r})>$ $-(n-k-2) / 2$. We assume that $\tau(\tilde{r})^{2} \leq \frac{(n-k-2)^{2}}{4}-2 \varepsilon$ where $\varepsilon>0$. Choose $\gamma:=\sqrt{\frac{(n-k-2)^{2}}{4}-\varepsilon}$. Using Step 3 we calculate

$$
\tau^{\prime}(r)=\frac{\omega^{\prime \prime}(r)}{\omega(r)}-\left(\frac{\omega^{\prime}(r)}{\omega(r)}\right)^{2} \geq \gamma^{2}-\tau(r)^{2}
$$

for all $r \geq \tilde{r}$, and thus $\tau^{\prime}(r) \geq \varepsilon$ as long as $\tau(r)^{2} \leq \frac{(n-k-2)^{2}}{4}-2 \varepsilon$. An easy argument on first-order ordinary differential equations yields an $R>\tilde{r}$ such 
that

$$
\tau(r) \geq \sqrt{\frac{(n-k-2)^{2}}{4}-2 \varepsilon},
$$

for all $r \geq R$. As $\varepsilon>0$ can be chosen arbitrarily small we conclude that (3.13a) must hold, and Step (3) follows.

Step 4. If (3.13a) holds then $L^{p_{n}}$-boundedness of $u$ contradicts the Assumption (3.2).

Since $\mathbb{M}_{-c}^{n, k}=\mathbb{M}_{c}^{n, k}$ we may assume that $c \geq 0$. At first we consider the case $c>0$. In the following argument, we denote by $C$ a positive constant that might change value from line to line. Since

$$
\mathrm{vol}^{g_{r}}\left(F_{r}\right)=\int_{F_{r}}\left(\frac{\sinh (c r)}{c}\right)^{k} d v^{\rho^{k}+\rho^{n-k-1}} \leq C \mathrm{e}^{k c r}
$$

we get

$$
\begin{aligned}
\int_{0}^{\infty} \omega(r)^{p_{n}} e^{-\frac{2 k c}{n-2} r} d r & =\int_{0}^{\infty}\left(\int_{F_{r}} u^{2} d v^{g_{r}}\right)^{\frac{p_{n}}{2}} \mathrm{e}^{-\frac{2 k c}{n-2} r} d r \\
& \leq C \int_{0}^{\infty} \int_{F_{r}} u^{p_{n}} d v^{g_{r}} d r \\
& \leq C \int_{\mathbb{M}_{c}^{n, k}} u^{p_{n}} d v^{G_{c}}
\end{aligned}
$$

using the Hölder inequality. This is bounded since $u$ is assumed to be in $L^{p_{n}}\left(\mathbb{M}_{c}^{n, k}\right)$. If (3.13a) holds then for any $\gamma \in\left(0, \frac{n-k-2}{2}\right)$ there is an $r_{1}=r_{1}(\gamma)$ so that

$$
\omega(r) \geq C \mathrm{e}^{\gamma r},
$$

for all $r \geq r_{1}$. Thus

$$
\int_{0}^{\infty} \omega(r)^{p_{n}} \mathrm{e}^{-\frac{2 k c}{n-2} r} d r \geq C \int_{r_{1}}^{\infty} \mathrm{e}^{b r} d r
$$

where

$$
b:=p_{n} \gamma-\frac{2 k c}{n-2}=\frac{2}{n-2}(n \gamma-k c) .
$$

If $b \geq 0$ then the right-hand side of (3.15) is infinite, which gives a contradiction to the boundedness of (3.14). Thus, we have $b<0$, implying $\gamma<k c / n$. Taking $\gamma \rightarrow(n-k-2) / 2$ yields $(n-k-2) / 2 \leq k|c| / n$, which finishes the proof of Step (3) for $c>0$.

The case $c=0$ can be solved with similar estimates. 
Step 5. Conclusion.

It remains to show the $L^{2}$-boundedness of $u$ if (3.13b) of Step 3 holds. We choose any $\gamma \in(0,(n-k-2) / 2)$. If (3.13b) holds we have

$$
\omega(r) \leq C \mathrm{e}^{-\gamma r}
$$

for all $r \geq r_{2}(\gamma)$, and by possibly enlarging $C$ this holds for all $r$. From this estimate, we have

$$
\int_{\mathbb{M}_{c}^{n, k}} u^{2} d v^{G_{c}}=\int_{0}^{\infty} \omega(r)^{2} d r<\infty
$$

which ends the proof of Theorem 3.1.

\section{A counterexample to Theorem 3.1 for $k=n-3$}

As noted after Theorem 3.1, Assumption (3.2) holds if $n \leq 6, k \in\{0, \ldots$, $n-3\},|c|<1$ or $n \geq 7, k \in\{0, \ldots, n-4\}$. It is natural to ask whether the conclusion of Theorem 3.1 holds for all $k \in\{0, \ldots, n-3\}$. The following proposition answers this in the negative.

Proposition 4.1. Let $n \geq 7$. There exists a smooth positive function $u \in L^{\infty}\left(\mathbb{H}_{1}^{n-2} \times \mathbb{S}^{2}\right) \cap L^{p_{n}}\left(\mathbb{H}_{1}^{n-2} \times \mathbb{S}^{2}\right)$ which satisfies

$$
L^{G_{1}} u=0
$$

and is not in $L^{2}\left(\mathbb{H}_{1}^{n-2} \times \mathbb{S}^{2}\right)$.

Note that the function $u$ given by Proposition 4.1 satisfies Inequality (3.1) with $\mu=0$.

Proof. Consider $\mathbb{S}^{n-3}$ as a totally geodesic sphere in $\mathbb{S}^{n}$. For $y \in \mathbb{S}^{n}$ let $\Gamma_{y}$ be the Green's function of $L^{\rho^{n}}$ at $y$. That is $\Gamma_{y}$ satisfies $L^{\rho^{n}} \Gamma_{y}=\delta_{y}$ in the sense of distributions, where $\delta_{y}$ is the Dirac distribution at $y$. It is well known that $\Gamma_{y}$ exists and satisfies $\Gamma_{y}(x) \sim\left(4(n-1) \omega_{n-1}\right)^{-1} r(x)^{-(n-2)}$ when $x$ tends to $y$. Here $r(x)$ denotes the geodesic distance from $x$ to $y$. Define $H$ on $\mathbb{S}^{n} \backslash \mathbb{S}^{n-3}$ by

$$
H(x):=\int_{\mathbb{S}^{n-3}} \Gamma_{y}(x) d v^{\rho^{n-3}}(y) .
$$

It is straightforward to check that for $x$ tending to $\mathbb{S}^{n-3}$ we have $H(x) \sim$ $c_{n}^{\prime} r^{\prime}(x)^{-1}$ where $c_{n}^{\prime}$ only depends on $n$ and where $r^{\prime}$ is the geodesic distance 
to $\mathbb{S}^{n-3}$. Hence, we have $H \in L^{p_{n}}\left(\mathbb{S}^{n} \backslash \mathbb{S}^{n-3}\right)$ since $n \geq 7$. In [4, Proposition 3.1] it was proven that $\mathbb{S}^{n} \backslash \mathbb{S}^{n-3}$ and $\mathbb{H}_{1}^{n-2} \times \mathbb{S}^{2}$ are conformal. Let $f$ be the conformal factor so that $G_{1}=f^{\frac{4}{n-2}} \rho^{n}$. As explained in [4], $f\left(r^{\prime}\right) \sim\left(r^{\prime}\right)^{-\frac{2}{n-2}}$ when $r^{\prime}$ tends to 0 . We set $u:=f^{-1} H$. By conformal covariance of the conformal Laplacian we have

$$
L^{G_{1}} u=0
$$

Moreover,

$$
\int_{\mathbb{H}_{1}^{n-2} \times \mathbb{S}^{2}} u^{p_{n}} d v^{G_{1}}=\int_{\mathbb{S}^{n} \backslash \mathbb{S}^{n-3}} H^{p_{n}} d v^{\rho^{n}}<\infty .
$$

In addition, using the asymptotics of $f$ given above, it is easy to check that $u$ is not in $L^{2}$ and hence provides the desired counterexample.

\section{Consequences for the surgery formula}

The goal of this paper is to find explicit lower bounds for $\Lambda_{n, k}$. We find the following.

Corollary 5.1. Assume that $k \in\{2, \ldots, n-4\}$, then

$$
\Lambda_{n, k} \geq \underline{\Lambda}_{n, k},
$$

where

$$
\underline{\Lambda}_{n, k}:=\frac{n a_{n}}{\left((k+1) a_{k+1}\right)^{\frac{k+1}{n}}\left((n-k-1) a_{n-k-1}\right)^{\frac{n-k-1}{n}}} \mu\left(\mathbb{S}^{k+1}\right)^{\frac{k+1}{n}} \mu\left(\mathbb{S}^{n-k-1}\right)^{\frac{n-k-1}{n}} .
$$

Note that we have $\Lambda_{n, 0}=\mu\left(\mathbb{S}^{n}\right)$ from [4, Section 3.5], and hence the only cases not covered by this corollary are $k=1$ and $k=n-3$. Further,

$$
\underline{\Lambda}_{n, 2}=\underline{\Lambda}_{n, n-4}=n a_{n}\left(\frac{\mu\left(\mathbb{S}^{3}\right)}{3 a_{3}}\right)^{\frac{3}{n}}\left(\frac{\mu\left(\mathbb{S}^{n-3}\right)}{(n-3) a_{n-3}}\right)^{\frac{n-3}{n}}=n a_{n}\left(\frac{\pi^{4}}{16}\right)^{\frac{1}{n}} \nu_{n-3}^{1 / n},
$$

where we defined

$$
\nu_{\ell}:=\left(\frac{\mu\left(\mathbb{S}^{\ell}\right)}{\ell a_{\ell}}\right)^{\ell}=\omega_{\ell}^{2}\left(\frac{\ell-2}{4}\right)^{\ell}
$$

and it holds that

$$
\omega_{\ell}=\operatorname{vol}\left(\mathbb{S}^{\ell}\right)=\frac{2 \pi^{(\ell+1) / 2}}{\Gamma\left(\frac{\ell+1}{2}\right)} .
$$




\begin{tabular}{c|ccc}
$n$ & $Y\left(\mathbb{S}^{n}\right)$ & $\underline{\Lambda}_{n, 2+}$ & $\underline{\lambda}_{n}$ \\
\hline 7 & 113.5272754 & 74.50435 & \\
8 & 130.7157953 & 92.24278367 & \\
9 & 147.8778709 & 109.4260421 & \\
10 & 165.0220642 & 126.4134026 & \\
11 & 182.1536061 & 143.3280094 & 135.9033973 \\
12 & 199.2758713 & 160.2189094 & 158.7256737 \\
13 & 216.3911332 & 177.1071517 & 178.0562033 \\
14 & 233.5009793 & 194.0019409 & 196.2714765 \\
15 & 250.6065514 & 210.9071013 & 213.9967504 \\
16 & 267.7086915 & 227.8239126 & 231.4689436 \\
17 & 284.8080344 & 244.7524346 & 248.7967717 \\
18 & 301.9050675 & 261.6921542 & 266.0365304
\end{tabular}

Figure 1: Some values for $\underline{\Lambda}_{n, 2+}$ and $\underline{\lambda}_{n}$.

We define

$$
\underline{\Lambda}_{n, 2+}:=\min \left\{\underline{\Lambda}_{n, 2}, \ldots, \underline{\Lambda}_{n, n-4}\right\} \text {. }
$$

Some values for $\underline{\Lambda}_{n, 2+}$ are listed in Figure 1. Numerically, we calculated $\underline{\Lambda}_{n, 2+}=\underline{\Lambda}_{n, 2}$ for $n \leq 3000$, and it seems reasonable to conjecture this for all $n$, but we do not have a proof.

Proof of Corollary 5.1. The conformal Yamabe invariant $\mu\left(\mathbb{M}_{c}^{n, k}\right)$ as defined in Section 2 for non-compact manifolds is, by virtue of [3, Theorem 2.3], bounded from below by $\underline{\Lambda}_{n, k}$. To see this we just have to notice that $\mu\left(\mathbb{H}_{c}^{k+1}\right)=\mu\left(\mathbb{S}^{k+1}\right)$, which holds since $\mathbb{H}_{c}^{k+1}$ is conformal to a subset of $\mathbb{S}^{k+1}$. Thus Corollary 5.1 is a direct consequence of Corollary 3.2.

\section{Topological applications}

In this section, we derive some topological consequences of our main theorem. Recall that by definition a manifold $M$ is $k$-connected, $k \geq 1$, if it is connected and if $\pi_{1}(M)=\pi_{2}(M)=\cdots=\pi_{k}(M)=0$.

Proposition 6.1. Let $M_{0}$ and $M_{1}$ be non-empty compact two-connected manifolds of dimension $n \geq 7$, and assume that $M_{0}$ is spin bordant to $M_{1}$. Then $M_{1}$ can be obtained from $M_{0}$ by a sequence of surgeries of dimensions $\ell, 3 \leq \ell \leq n-4$.

Note that two-connected manifolds are orientable and spin, and they carry a unique spin structure. 
The proposition is well known, but for the sake of being self-contained we include a proof following the lines of [10, Lemma 4.2]). As a first step we prove a lemma.

Lemma 6.2. Let $M_{0}$ and $M_{1}$ compact spin manifolds of dimension $n \geq 7$ and assume that $M_{0}$ is spin bordant to $M_{1}$. Then there is a three-connected spin bordism $W$ from $M_{0}$ to $M_{1}$.

Proof of the Lemma. We start with a given spin bordism $W_{0}$ from $M_{0}$ to $M_{1}$. From this bordism we construct a bordism $W$ that is three-connected.

By performing 0-dimensional surgeries at the bordism, one can modify the original bordism $W_{0}$ to be connected. This can be done such that the bordism $W_{1}$ thus obtained is again orientable, and $W_{1}$ then carries a spin structure.

We now perform one-dimensional surgeries to reduce the fundamental group to the trivial group. Assume that $[\gamma] \in \pi_{1}\left(W_{1}\right)$. We can assume that $\gamma: S^{1} \rightarrow W_{1}$ is an embedding. Its normal bundle is trivial as $W_{1}$ is orientable. Performing a one-dimensional surgery along $\gamma$ using a trivialization $\nu$ of this normal bundle yields a new bordism $W_{1}^{\gamma, \nu}$ which depends both on $\gamma$ and $\nu$. This bordism is orientable. For any $\gamma$ one can choose a trivialization $\nu$ such that the bordism $W_{1}^{\gamma, \nu}$ carries a spin structure that coincides with the spin structure of $W_{1}$ outside a tubular neighborhood of the image of $\gamma$. The van Kampen Theorem gives a surjective homomorphism $\pi_{1}\left(W_{1}\right) \rightarrow$ $\pi_{1}\left(W_{1}^{\gamma, \nu}\right)$ such that $[\gamma]$ is in the kernel. The fundamental group $\pi_{1}(W)$ is finitely generated, let $\gamma_{i}$ be disjoint embedded circles such that $\left[\gamma_{1}\right], \ldots,\left[\gamma_{\ell}\right]$ is a set of generators $\pi_{1}(W)$. Performing one-dimensional surgeries along the $\gamma_{i}$ with suitable trivializations of their normal bundles then yields a simply connected spin bordism $W_{2}$ from $M_{0}$ to $M_{1}$.

Next we perform two-dimensional surgeries to remove $\pi_{2}\left(W_{2}\right)$. Assume that $[\sigma] \in \pi_{2}\left(W_{2}\right)$ is given and assume that $\sigma: S^{2} \rightarrow W_{2}$ is an embedding. Since $W_{2}$ is spin the normal bundle of the image of $\sigma$ is trivial. Performing a two-dimensional surgery along $\sigma$ yields a new spin bordism $W_{2}^{\sigma}$ which depends on the choice of $\sigma$. However, it is independent of the choice of trivialization as different trivializations are homotopic. After a finite number of two-dimensional surgeries we obtain a two-connected spin bordism $W_{3}$ from $M_{0}$ to $M_{1}$.

In a similar way, one can also remove $\pi_{3}\left(W_{3}\right)$. The Whitney embedding theorem implies that any class in $\pi_{3}\left(W_{3}\right)$ can be represented by an embed$\operatorname{ding} \tau: S^{3} \rightarrow W_{3}$, as $n \geq 6$. The normal bundle of the image of $\tau$ is trivial, as $\pi_{2}(O(n-3))=0$. A surgery along $\tau$ with any trivialization $\nu$ will then 
kill $[\tau]$, and since $n \geq 7$ the spin bordism $W_{3}^{\tau, \nu}$ thus obtained is again twoconnected and will have $\pi_{3}\left(W_{3}^{\tau, \nu}\right) \cong \pi_{3}\left(W_{3}\right) /[\tau]$. After finitely many surgery steps we obtain a three-connected bordism $W$ as claimed in the lemma.

Proof of the Proposition. Assume that $W$ is three-connected spin bordism from $M_{0}$ to $M_{1}$. Then $H_{i}\left(W, M_{j}\right)=0$ for $i=0,1,2,3$, in particular $b_{i}\left(W, M_{0}\right)=0$ for these numbers $i$. We can apply [9, VIII Theorem 4.1] for $k=4$ and $m=n+1$. One obtains that there is a presentation of the bordism such that for any $i<4$ and any $i>n-3$ the number of $i$-handles is given by $b_{i}\left(W, M_{0}\right)$. Any $i$-handle corresponds to a surgery of dimension $i-1$. It remains to show that $b_{i}\left(W, M_{0}\right)=0$ for $i \in\{0,1,2,3, n+1, n, n-$ $1, n-2\}$. For $i \in\{0,1,2,3\}$ this was discussed above. By Poincaré duality $H^{n+1-i}\left(W, M_{0}\right)$ is dual to $H_{i}\left(W, M_{1}\right)$ which vanishes for $i=0,1,2,3$. On the other hand, the universal coefficient theorem tells us that the free parts of $H^{i}\left(W, M_{0}\right)$ and $H_{i}\left(W, M_{0}\right)$ are isomorphic. Thus $b_{i}\left(W, M_{0}\right)$ that is by definition the rank of (the free part of) $H_{i}\left(W, M_{0}\right)$ vanishes for $i \in$ $\{n+1, n, n-1, n-2\}$.

Corollary 6.3. Let $M$ be a two-connected compact manifold of dimension $n \geq 7$ which is a spin boundary. Then

$$
\sigma(M) \geq \underline{\Lambda}_{n, 2+},
$$

where $\underline{\Lambda}_{n, 2+}$ is defined in Section 5 .

Proof. Assume that $M$ is the boundary of a compact spin manifold $W$ of dimension $n+1$. By removing a ball we obtain a spin-bordism from $S^{n}$ to $M$. The preceding proposition tells us that $M$ can be obtained by surgeries of dimensions $\ell \in\{3, \ldots, n-4\}$ from $S^{n}$. By applying the surgery formula (1.1) and Corollary 5.1 we get the stated lower bound for $\sigma(M)$.

Theorem 6.4 Stolz [14, Theorem B]. Let $M$ be a compact spin manifold of dimension $n \geq 5$. Assume that the index $\alpha(M) \in K O_{n}(\mathrm{pt})$ vanishes. Then $M$ is spin-bordant to the total space of an $\mathbb{H} P^{2}$-bundle over a base $Q$ for which the structure group is $\operatorname{PSp}(3)$.

The base $Q$ has to be understood as a spin manifold, so that it admits a spin structure and the choice of spin structure matters. The theorem includes the fact the every spin manifold of dimension 5,6 , or 7 is a spin boundary, in these cases $Q=\emptyset$. 
Proposition 6.5 (Extended Stolz theorem). In the case $n \geq 9$ one can assume that the base $Q$ is connected, and in the case $n \geq 11$ one can assume that it is simply connected.

Note that $M:=\mathbb{H} P^{2} \amalg \mathbb{H} P^{2}$ is an example of an eight-dimensional manifold where $Q$ cannot be chosen to be connected. This follows from the fact that $\mathbb{H} P^{2}$ has non-vanishing signature and thus $\left[\mathbb{H} P^{2}\right]$ is an element of infinite order in $\Omega_{8}^{\text {spin }}$. If $S^{1}$ carries the spin structure that does not bound a disc, then $\mathbb{H} P^{2} \times S^{1}$ and $\mathbb{H} P^{2} \times S^{1} \times S^{1}$ are examples of dimensions 9 and 10 where $Q$ cannot be chosen to be simply connected. This is a consequence of the fact that $\left[\mathbb{H} P^{2} \times S^{1}\right] \in \Omega_{9}^{\text {spin }}$ and $\left[\mathbb{H} P^{2} \times S^{1} \times S^{1}\right] \in \Omega_{10}^{\text {spin }}$ are non-zero elements (of order 2), see [6, Corollary 1.9] or [7, Corollary 2.6].

Proof. Assume that $M$ is spin bordant to a spin manifold $N_{0}$ which is the total space of a fibre bundle with fibre $\mathbb{H} P^{2}$ and structure group PSp(3) over a base $Q_{0}$ of dimension $n-8 \geq 1$. By performing zero-dimensional surgery on $Q_{0}$ we obtain a connected space $Q_{1}$. The spin bordism from $Q_{0}$ to $Q_{1}$ which is associated to the zero-dimensional surgeries yields a spin bordism from $N_{0}$ to a total space of a fibre bundle with fibre $\mathbb{H} P^{2}$ and structure group $\operatorname{PSp}(3)$ over $Q$. This $Q$ is connected, but not necessarily simply connected.

Now assume that $n \geq 11$. Any path $\gamma: S^{1} \rightarrow Q_{1}$ is homotopic to an embedding and has a trivial normal bundle as $Q_{1}$ is orientable. A tubular neighbourhood of the image of $\gamma$ in $Q_{1}$ is diffeomorphic to $S^{1} \times B^{n-9}$. Any trivialization of this normal bundle yields the germ of such a diffeomorphism up to isotopy. Because of our condition $n \geq 11$, we can choose the trivialization of the normal bundle such that the induced spin structure on $S^{1} \times B^{n-9}$ is the bounding spin structure of $B^{2} \times B^{n-9}$. Doing a surgery along $\gamma$ with respect to such a trivialization we obtain a spin manifold $Q_{2}$, and the associated bordism from $Q_{1}$ to $Q_{2}$ is a spin bordism. As $\operatorname{PSp}(3)$ is connected the $\mathbb{H} P^{2}$-bundle with structure group PSp(3) extends to a bundle of the same type over this bordism.

We now perform a sequence of such one-dimensional surgeries, where $\gamma$ runs through a generating set of $\pi_{1}\left(Q_{1}\right)$. The space thus obtained is then simply connected.

Combining the previous results we obtain the following.

Corollary 6.6. Let $M$ be a two-connected compact manifold of dimension $n=7$. Then

$$
\sigma(M) \geq \underline{\Lambda}_{7,2+}>74.5
$$


To derive a similar result for $n=8$, we remark that the conformal Yamabe constant of $\mathbb{H} P^{2}$, equipped with the standard metric, is $128 \pi / 120^{1 / 4}=$ $121.4967 \ldots>\underline{\Lambda}_{8,2+}=92.24278 \ldots$

Corollary 6.7. Let $M$ be a two-connected compact manifold of dimension $n=8$. Then $\sigma(M)=0$ if $\alpha(M) \neq 0$, and

$$
\sigma(M) \geq \underline{\Lambda}_{8,2+}>92.2
$$

if $\alpha(M)=0$.

Proposition 6.8. Let $M_{0}$ be the total space of a bundle with fibre $\mathbb{H} P^{2}$ and structure group $\mathrm{PSp}(3)$ over a base $B$ of dimension $n-8$. Then, if $n \geq 11$

$$
\sigma\left(M_{0}\right) \geq \underline{\lambda}_{n}:=n a_{n}\left(\frac{3^{6} 2^{18}}{7^{8} 5^{2}} \pi^{8}\right)^{1 / n} \nu_{n-8}^{1 / n}
$$

Proof. M. Streil has shown in [15] that $\sigma\left(M_{0}\right) \geq \mu\left(\mathbb{H} P^{2} \times \mathbb{R}^{n-8}\right)$, where $\mathbb{H} P^{2} \times \mathbb{R}^{n-8}$ carries the product metric of the standard metrics on $\mathbb{H} P^{2}$ and $\mathbb{R}^{n-8}$. On the other hand, it follows from [3, Theorem 2.3] that

$$
\mu\left(\mathbb{H} P^{2} \times \mathbb{R}^{n-8}\right) \geq \frac{n a_{n}}{\left(8 a_{8}\right)^{8 / n}\left((n-8) a_{n-8}\right)^{(n-8) / n}} \mu\left(\mathbb{H} P^{2}\right)^{8 / n} \mu\left(\mathbb{S}^{n-8}\right)^{(n-8) / n} .
$$

We also have

$$
\left(\frac{\mu\left(\mathbb{H} P^{2}\right)}{8 a_{8}}\right)^{8}=\frac{3^{6} 2^{18}}{7^{8} 5^{2}} \pi^{8}=1.32599 \ldots \pi^{8}=12581.78 \ldots
$$

This clearly implies the proposition.

As an example we study $n=11$. Then $\nu_{3}=\pi^{6} / 8$ and thus $\underline{\lambda}_{11}=178.23277$. Some further values for $\underline{\lambda}_{n}$ are listed in figure 1 .

Proposition 6.9. Let $M$ be a two-connected compact manifold of dimension $n \geq 11$. Then $\sigma(M)=0$ if $\alpha(M) \neq 0$. If $\alpha(M)=0$, then

$$
\sigma(M) \geq \begin{cases}\underline{\lambda}_{11}>135.90, & \text { if } n=11 \\ \underline{\lambda}_{12}>158.72, & \text { if } n=12 \\ \underline{\Lambda}_{n, 2+}, & \text { if } n \geq 13\end{cases}
$$


Proof. If $\alpha(M)=0$, then we have seen

$$
\sigma(M) \geq \min \left(\underline{\Lambda}_{n, 2+}, \underline{\lambda}_{n}\right)
$$

It remains to compare $\underline{\Lambda}_{n, 2+}$ and $\underline{\lambda}_{n}$. Numerically, we calculated $\underline{\lambda}_{11} \leq$ $\underline{\Lambda}_{11,2+}, \underline{\lambda}_{12} \leq \underline{\Lambda}_{12,2+}$, and $\underline{\lambda}_{n} \geq \underline{\Lambda}_{n, 2+}$ for $13 \leq n \leq 5000$.

For $n \geq 1100$, we found

$$
\underline{\lambda}_{n}^{n} \geq 1.43 \underline{\Lambda}_{n, 2}^{n} \geq 1.43 \underline{\Lambda}_{n, 2+}^{n}
$$

by studying the $\Gamma$-function and by using $\Gamma(n) / \Gamma(n-1 / 2) \geq \sqrt{n-1}$. This yields the required result.

\section{Appendix A. Details of an argument}

We here give some details on an argument sketched directly after Question 2.1.

Proposition A.1. Let $(N, h)$ be a complete $n$-dimensional Riemannian manifold of bounded geometry and let $u \in L^{2}(N) \cap L^{p_{n}}(N)$ be a positive solution of the Yamabe equation (2.3). Then $\int_{N} u \Delta^{h} u d v^{h}$ and $\int_{N}|d u|^{2} d v^{h}$ exist and satisfy

$$
\int_{N} u \Delta^{h} u d v^{h}=\int_{N}|d u|^{2} d v^{h}
$$

It also follows that $u \in H_{1}^{2}(N)$ and $\mu \geq \mu(N, h)$.

Proof. Since $u L^{h} u=\mu u^{p_{n}}$ it is clear that $\int u L^{h} u d v^{h}$ exists and as $s^{h}$ is bounded and because of $u \in L^{2}$ this also implies the existence of the integral $\int_{N} u \Delta^{h} u d v^{h} \in \mathbb{R}$.

Let $\eta_{i}: N \rightarrow[0,1]$ be a sequence of smooth compactly supported cut-off functions such that $\left|d \eta_{i}\right| \leq 1$ and such that the $\eta_{i} \equiv 1$ on $K_{i}$ where $K_{i}$ is a compact exhaustion of $N$, that is $N=\bigcup K_{i}$. In particular, $\int_{\operatorname{supp}} d \eta_{i} u^{2} d v^{h} \rightarrow$ 0 for $i \rightarrow \infty$. We can also assume that $\eta_{i+1} \geq \eta_{i}$.

In the case $(N, h)=\mathbb{M}_{c}^{n, k}$ such functions can be obtained as $\eta_{i}(x):=$ $\chi(r(x)-2 i)$ where $r$ is the radial function on $\mathbb{H}_{c}^{k+1}$ and where $\chi: \mathbb{R} \rightarrow$ $[0,1]$ is a smooth function with $0 \leq \chi^{\prime} \leq 1,\left.\chi\right|_{(-\infty, 0]} \equiv 0,\left.\chi\right|_{[2, \infty)} \equiv 1$. For general $(N, h)$ of bounded geometry, this existence follows similarly using $[13$, Appendix A1.2., Lemma 2.1., page 70]. 
We calculate

$$
\begin{aligned}
\int_{N}\left|d\left(\eta_{i} u\right)\right|^{2} d v^{h} & =\int_{N}\left(u^{2}\left|d \eta_{i}\right|^{2}+\eta_{i} u\left\langle d \eta_{i}, d u\right\rangle+\left\langle d u, \eta_{i} d\left(\eta_{i} u\right)\right\rangle\right) d v^{h} \\
& =\int_{N}\left(u^{2}\left|d \eta_{i}\right|^{2}+\eta_{i} u\left\langle d \eta_{i}, d u\right\rangle+\left\langle d u, d\left(\eta_{i}^{2} u\right)-\eta_{i} u d \eta_{i}\right\rangle\right) d v^{h} \\
& =\int_{N}\left(u^{2}\left|d \eta_{i}\right|^{2}+\left\langle d u, d\left(\eta_{i}^{2} u\right)\right\rangle\right) d v^{h} \\
& =\int_{N} u^{2}\left|d \eta_{i}\right|^{2} d v^{h}+\int_{N} \eta_{i}^{2} u \Delta^{h} u d v^{h} .
\end{aligned}
$$

As $u$ is in $L^{p_{n}} \cap L^{2}$ and as $s^{h}$ is bounded, we see that

$$
\left(1-\eta_{i}^{2}\right) u \Delta^{h} u=\left(1-\eta_{i}^{2}\right) \underbrace{\left(\frac{\mu}{a_{n}} u^{p_{n}}-\frac{s^{h}}{a_{n}} u^{2}\right)}_{\in L^{1}}
$$

converges to 0 in $L^{1}$. Thus $\int_{N} \eta_{i}^{2} u \Delta^{h} u d v^{h}$ exists and it converges to $\int_{N} u \Delta^{h} u d v^{h}$.

We also see that

$$
\int_{N} u^{2}\left|d \eta_{i}\right|^{2} d v^{h} \leq \int_{\operatorname{supp} d \eta_{i}} u^{2} d v^{h} \rightarrow 0
$$

Thus,

$$
\int_{N}\left|d\left(\eta_{i} u\right)\right|^{2} d v^{h} \rightarrow \int_{N} u \Delta^{h} u d v^{h}
$$

and together with $\left\|\eta_{i} u\right\|_{L^{p_{n}}} \rightarrow\|u\|_{L^{p_{n}}}=1$ this implies

$$
\mathcal{F}^{g}\left(\eta_{i} u\right) \rightarrow \mu
$$

from which we directly see that $\mu \geq \mu(N, h)$.

From (A.1) we also see $\int_{N} u \Delta^{h} u d v^{h} \geq 0$ and (A.1) can be rewritten as

$$
\left\|d\left(\eta_{i} u\right)\right\|_{L^{2}} \rightarrow \sqrt{\int_{N} u \Delta^{h} u d v^{h}}
$$


As a consequence

$$
\begin{aligned}
\left\|\eta_{i} d u\right\|_{L^{2}(N)} & \leq\left\|d\left(\eta_{i} u\right)\right\|_{L^{2}(N)}+\left\|u d \eta_{i}\right\|_{L^{2}(N)} \\
& \leq\left\|d\left(\eta_{i} u\right)\right\|_{L^{2}(N)}+\|u\|_{L^{2}\left(\operatorname{supp} d \eta_{i}\right)} \\
& \rightarrow \sqrt{\int_{N} u \Delta^{h} u d v^{h}}
\end{aligned}
$$

is bounded. As $\eta_{i}|d u|$ is increasing with limit $|d u|$, we get $|d u| \in L^{2}$ with $\|d u\|_{L^{2}} \leq \sqrt{\int_{N} u \Delta^{h} u d v^{h}}$, and thus $u \in H_{1}^{2}$.

On the other hand,

$$
\left\|d\left(\eta_{i} u\right)\right\|_{L^{2}(N)} \leq \underbrace{\|u\|_{L^{2}\left(\operatorname{supp} d \eta_{i}\right)}}_{\rightarrow 0}+\|d u\|_{L^{2}(N)},
$$

so

$$
\int_{N}|d u|^{2} d v^{h}=\|u\|_{L^{2}(N)}^{2}=\int_{N} u \Delta^{h} u d v^{h} .
$$

\section{Acknowledgments}

B. Ammann was partially supported by the DFG Sachbeihilfe AM 144/2-1. M. Dahl was partially supported by the Swedish Research Council. E. Humbert was partially supported by ANR-10-BLAN 0105. We want to thank J. Petean for enlightening discussions related to this article. We thank N. Große for interesting discussions on partial differential equations on non-compact manifolds of bounded geometry and for showing us the reference [13]. Further, we thank the anonymous referee for comments that helped us improve the paper in several ways.

\section{References}

[1] B. Ammann, M. Dahl, and E. Humbert, Surgery and the spinorial $\tau$-invariant, Comm. Part. Diferf. Eq. 34 (2009), 1147-1179.

[2] _ Low-dimensional surgery and the Yamabe invariant, Preprint to appear in J. Math. Soc. Japan, 2012; http://arXiv.org/ abs/1204.1197, available on http://mathsoc.jp/publication/JMSJ/ inpress.html. 
[3] The conformal Yamabe constant of product manifolds, Proc. AMS 141 (2013), 295-307.

[4] Smooth Yamabe invariant and surgery, J. Differ. Geom. 94 (2013), 1-58.

[5] B. Ammann and N. Große, Relations between threshold constants for Yamabe type bordism invariants, in preparation.

[6] D.W. Anderson, E.H. Brown, Jr. and F.P. Peterson, Spin cobordism, Bull. Amer. Math. Soc. 72 (1966), 256-260.

[7] , The structure of the Spin cobordism ring, Ann. Math. 86(2) (1967), 271-298.

[8] O. Kobayashi, Scalar curvature of a metric with unit volume, Math. Ann. 279 (1987), 253-265.

[9] A.A. Kosinski, Differential manifolds, in 'Pure and Applied Mathematics', vol. 138, Academic Press, Boston, MA, 1993.

[10] M.-L. Labbi, Stability of the p-curvature positivity under surgeries and manifolds with positive Einstein tensor, Ann. Global Anal. Geom. 15(4) (1997), 299-312.

[11] J. Petean and J.M. Ruiz, Isoperimetric profile comparisons and Yamabe constants, Ann. Global Anal. Geom. 40 (2011), 177-189.

[12] On the Yamabe constants of $S^{2} \times \mathbb{R}^{3}$ and $S^{3} \times \mathbb{R}^{2}$, Differ. Geom. Appl. 31 (2013), 308-319.

[13] M.A. Shubin, Spectral theory of elliptic operators on noncompact manifolds, Astérisque (1992), no. 207, 35-108, Méthodes semi-classiques, Vol. 1 (Nantes, 1991).

[14] S. Stolz, Simply connected manifolds of positive scalar curvature, Ann. Math. (2) 136(3) (1992), 511-540.

[15] M. Streil, PhD thesis, in preparation, University of Regensburg. 
FAKULTät FÜR MATHEMATIK

UNIVERSITÄT REGENSBURG

93040 REgENSBURG

GERMANY

E-mail address: bernd.ammann@mathematik.uni-regensburg.de

INSTITUTIONEN FÖR MATEMATIK

Kungliga TeKNiska HÖGSKOLAN

10044 Stockholm

SWEDEN

E-mail address: dahl@math.kth.se

Laboratoire de Mathématiques et Physique Théorique

Université DE TOURS

PARC DE GRANDMONT

37200 TOURS

FRANCE

E-mail address: Emmanuel.Humbert@lmpt.univ-tours.fr

Received November 17, 2011 